\title{
Degenerate Svinolupov KdV systems
}

\author{
Metin Gürses ${ }^{\text {a }}$, Atalay Karasu ${ }^{b}$ \\ a Department of Mathematics, Faculty of Sciences, Bilkent University, 06533 Ankara, Turkey \\ ' Department of Physics, Faculty of Arts and Sciences, Middle East Technical University, 0653I Ankara, Turkey \\ Received 8 January 1996; accepted for publication 14 February 1996 \\ Communicated by A.P. Fordy
}

\begin{abstract}
We find infinitely many coupled systems of $\mathrm{KdV}$ type equations which are integrable. We give also their recursion operators.
\end{abstract}

Recently, Svinolupov [1] has introduced a class of integrable multicomponent KdV equations associated with Jordan algebras ( JKdV). He has found a one-to-one correspondence between Jordan algebras and multicomponent $\mathrm{KdV}$ equations that possess infinitely many higher symmetries. In this work we extend his work on $\mathrm{KdV}$ systems to a more general form. In addition to the Jordan algebra related KdV systems found by Svinolupov $[1,2]$ we find new integrable systems of equations.

We consider a system of $N$ nonlinear equations of the form

$$
q_{t}^{i}=b_{j}^{i} q_{x x x}^{j}+s_{j k}^{i} q^{j} q_{x}^{k}
$$

where $i, j, k=1,2, \ldots, N, q^{i}$ are real and depend on the variables $x$ and $t, s_{j k}^{i}$ and $b_{j}^{i}$ are constants. We assume that the recursion operator of this system is given by

$$
R_{j}^{i}=b_{j}^{i} D^{2}+a_{j k}^{i} q^{k}+c_{j k}^{i} q_{x}^{k} D^{-1}+F_{l k j}^{i} q^{l} D^{-1} q^{k} D^{-1}
$$

where $a_{j k}^{i}, c_{j k}^{i}$ and $F_{l k j}^{i}$ arc constants with

$$
s_{j k}^{i}=a_{k j}^{i}+c_{j k}^{i}, \quad F_{l k j}^{i}=-F_{l j k}^{i} \text {. }
$$

The main purpose of this work is to find integrable subclasses of (1). In these classes the major problem is to determine $a_{j k}^{i}, c_{j k}^{i}$ and $F_{l k j}^{i}$ in terms of $b_{j}^{i}$ and $s_{j k}^{i}$ and to find the conditions satisfied by $b_{j}^{i}$ and $s_{j k}^{i}$ (integrability conditions).

The recursion operator $R_{j}^{i}$ satisfies the compatibility condition

$$
R_{j, t}^{i}=F_{k}^{i} R_{j}^{k}-R_{k}^{i} F_{j}^{\prime k}
$$


where $F_{k}^{\prime i}$ comes from the Fréchet derivative of system (1), which is given by

$$
\sigma_{t}^{i}=F_{j}^{i} \sigma^{j}
$$

where the $\sigma^{i}$ are called the symmetries of system (1). Eq. (5) is called the symmetry equation of (1), with

$$
F_{j}^{\prime i}=b_{j}^{i} D^{3}+s_{j k}^{i} q_{x}^{k}+s_{k j}^{i} q^{k} D
$$

Recursion operators are defined as operators mapping symmetries to symmetries, i.e.

$$
R_{j}^{i} \sigma^{i}=\lambda \sigma^{i}
$$

where $\lambda$ is an arbitrary constant. Eqs. (5) and (7) imply (4). It is Eq. (4) which determines the constants $a_{j k}^{i}, c_{j k}^{i}$ and $F_{l k j}^{i}$ in terms of the $b_{j}^{i}$ and $s_{j k}^{i}$. The same equation (4) brings severe constraints on the $b_{j}^{i}$ and $s_{j k}^{i}$.

We have two exclusive cases depending upon the matrix $b_{j}^{i}$. These are the nondegenerate Svinolupov system where $\operatorname{det}\left(b_{j}^{i}\right) \neq 0$ and the degenerate Svinolupov system where $\operatorname{det}\left(b_{j}^{i}\right)=0$. Our major result in this work is the degenerate Svinolupov KdV system.

(I) Nondegenerate Svinolupov KdV system. $\operatorname{det}\left(b_{j}^{i}\right) \neq 0$. In this case the constant parameters $a_{j k}^{i}, c_{j k}^{i}, s_{j k}^{i}$ are symmetric with respect to the subindices and $a_{j k}^{i}, c_{j k}^{i}$ are given by

$$
a_{j k}^{i}=\frac{2}{3} s_{j k}^{i}, \quad c_{j k}^{i}=\frac{1}{3} s_{j k}^{i},
$$

where $h_{j}^{i}$ and $s_{j k}^{i}$ have to satisfy the following constraints,

$$
\begin{aligned}
& b_{l}^{k} s_{j k}^{i}=b_{j}^{k} s_{k l}^{i}, \\
& s_{p r}^{k} F_{l j k}{ }^{i}+s_{j r}^{k} F_{l p k}^{i}+s_{j p}^{k} F_{l r k}^{i}=0,
\end{aligned}
$$

with

$$
F_{l^{l} j}^{r}=\frac{1}{9} C_{i}^{r}\left(s_{j k}^{i} s_{l p}^{k}-s_{l k}^{i} s_{j p}^{k}\right),
$$

and $C_{i}^{r}$ is the inverse of $b_{r}^{i}$. Eq. (10) is the equation satisfied by the structure constants of the Jordan algebra [1]. We now consider some particular cases.

(i) If $F=0$, we have the following equations,

$$
a_{j k}^{i}=\frac{2}{3} s_{j k}^{i}, \quad c_{j k}^{i}=\frac{1}{3} s_{j k}^{i},
$$

where $b_{j}^{i}$ and $s_{j k}^{i}$ satisfy

$$
\begin{aligned}
& s_{j k}^{i} s_{l p}^{k}-s_{l k}^{i} s_{j p}^{k}=0, \\
& b_{l}^{k} s_{j k}^{i}-b_{k}^{i} s_{j l}^{k}=0 .
\end{aligned}
$$

The recursion operator of this class is given by

$$
R_{j}^{i}=b_{j}^{i} D^{2}+\frac{2}{3} s_{j k}^{i} q^{k}+{ }_{3}^{1} s_{j k}^{i} q_{x}^{k} D^{-1} .
$$

At this point we assume that the $q^{i}$ are real and hence divide this class into two subcases. For the complex case such a division is irrelevant.

(a) If $b_{j}^{i}$ is diagonalizable then the system in (1) decouples because the Jordan algebra becomes associative as well as commutative [5]. 
(b) If $b_{j}^{i}$ is nondiagonalizable then we obtain distinct coupled systems. Let us consider the case where $N=2$. Solving the constraint equations (13) and (14) we obtain the following integrable system,

$$
\begin{aligned}
& u_{t}=v_{x x x}+r u_{x}-s v_{x}, \\
& v_{t}=-u_{x x x}+r v_{x}+s u_{x},
\end{aligned}
$$

where $r=c_{0} u+c_{1} v$ and $s=-c_{1} u+c_{0} v$. The recursion operator for the above system is given by

$$
R=\left(\begin{array}{cc}
\frac{2}{3} r+\frac{1}{3} r_{x} D^{-1} & D^{2}-\frac{2}{3} s-\frac{1}{3} s_{x} D^{-1} \\
-D^{2}+\frac{2}{3} s+\frac{1}{3} s_{x} D^{-1} & \frac{2}{3} r+\frac{1}{3} r_{x} D^{-1}
\end{array}\right) .
$$

In terms of the variables $r$ and $s$ the system of equations (16), (17) become

$$
\begin{aligned}
& r_{t}=s_{x x x}+r r_{x}-s s_{x}, \\
& s_{t}=-r_{x x x}+(r s)_{x} .
\end{aligned}
$$

This system is nothing but the complex KdV equation $\mathrm{i} \rho_{t}=\rho_{x x x}-\rho \rho_{x}$ with $\rho=\mathrm{i} r-s$.

(ii) If $F \neq 0$, we obtain the Jordan KdV systems introduced hy Svinolupov [1] with the following equations,

$$
a_{j k}^{i}=\frac{2}{3} s_{j k}^{i}, \quad c_{j k}^{i}=\frac{1}{3} s_{j k}^{i},
$$

where $b_{j}^{i}$ and $s_{j k}^{i}$ satisfy the following constraints,

$$
\begin{aligned}
& b_{l}^{k} s_{j k}^{i}=b_{k}^{i} s_{j l}^{k}, \\
& F_{p l i}{ }^{r}=\frac{1}{9} C_{i}^{r}\left(s_{j k}^{i} s_{l p}^{k}-s_{l k}^{i} s_{j p}^{k}\right), \\
& s_{p,{ }^{k}{ }_{l j k}{ }^{i}{ }^{i}+s_{j r}^{k} F_{l p k}{ }^{i}+s_{j p}^{k} F_{l r k}^{i}=0 .}^{i}=0
\end{aligned}
$$

The recursion operator becomes

$$
R_{j}^{i}=b_{j}^{i} D^{2}+\frac{2}{3} s_{j k}^{i} q^{k}+\frac{1}{3} s_{j k}^{i} q_{x}^{k} D^{-1}+\frac{1}{9} C_{r}^{i}\left(s_{j m}^{r} s_{k l}^{m}-s_{k m}^{r} s_{j l}^{m}\right) q^{l} D^{-1} q^{k} D^{-1} .
$$

Here there is only one choice $b_{j}^{i}=b_{0} \delta_{j}^{i}$ where $b_{0}$ can be taken as unity without losing any generality. The special cases $N=2$ and $N=3$ are given in Refs. [1,2].

(II) Degenerate Svinolupov KdV system. $\operatorname{det}\left(b_{j}^{i}\right)=0$ or $b_{j}^{i}$ is singular. Here we consider only the case where $F_{p l j}{ }_{j}=0$ and in addition we assume that the rank of the matrix $b_{j}^{i}$ is $N-1$. In this case we may take $b_{j}^{i}=\delta_{j}^{i}-k^{i} k_{j}$, where $k_{i}$ is a unit vector, $k^{i} k_{i}=1$. In this work we use the Einstein convention, i.e., repeated indices are summed up from 1 to $N$. We then have the following solution for all $N$,

$$
\begin{aligned}
& a_{k j}^{i}=\frac{2}{3} s_{j k}^{i}+\frac{1}{3}\left[k^{i}\left(k_{j} n_{k}-2 k_{k} n_{j}\right)+k_{k} k_{j} b^{i}\right], \\
& c_{j k}^{i}=\frac{1}{3} s_{j k}^{i}-\frac{1}{3}\left[k^{i}\left(k_{j} n_{k}-2 k_{k} n_{j}\right)+k_{k} k_{j} b^{i}\right],
\end{aligned}
$$

where

$$
\begin{aligned}
& n_{l}=k_{i} k^{j} s_{l j}^{i}, \\
& b^{i}=k^{j} k^{l} s_{l j}^{i} .
\end{aligned}
$$

The vectors $k^{i}$ and $s_{j k}^{i}$ are not arbitrary, they satisfy the following constraints,

$$
s_{j k}^{i} s_{l m}^{k}-s_{l k}^{i} s_{j m}^{k}=2\left(k_{j} n_{l}-k_{l} n_{j}\right)\left(-k^{i} n_{m}+b^{i} k_{m}\right),
$$




$$
\begin{aligned}
& k^{i} n_{i}=0, \\
& s_{j k}^{i}=s_{k j}^{i} .
\end{aligned}
$$

The recursion operator is given by

$$
\begin{aligned}
R_{j}^{i} & =b_{j}^{i} D^{2}+\left\{\frac{2}{3} s_{j k}^{i}+\frac{1}{3}\left[k^{i}\left(k_{j} n_{k}-2 k_{k} n_{j}\right)+k_{k} k_{j} b^{i}\right]\right\} q^{k} \\
& +\left\{\frac{1}{3} s_{j k}^{i}-\frac{1}{3}\left[k^{i}\left(k_{j} n_{k}-2 k_{k} n_{j}\right)+k_{k} k_{j} b^{i}\right]\right\} q_{x}^{k} D^{-1} .
\end{aligned}
$$

The first generalised symmetry is found as

$$
\begin{aligned}
& \frac{\partial q^{i}}{\partial \tau}=R_{j}^{i} q_{t}^{j}=b_{m}^{i} q_{x x x x x}^{m}+b_{j}^{i} s_{m n}^{j}\left(q^{m} q_{x x x}^{n}+3 q_{x}^{m} q_{x x}^{n}\right)+\frac{2}{3} b_{m}^{j} s_{k j}^{i} q^{k} q_{x x x}^{m}+\frac{1}{3} b_{m}^{j} s_{j k}^{i} q_{x}^{k} q_{x x}^{m} \\
& \quad+\frac{2}{3} s_{k j}^{i} s_{n n}^{j} q^{k} q^{m} q_{x}^{n}+\frac{1}{6} s_{j k}^{i} s_{m n}^{j} q_{x}^{k} q^{m} q^{n}+\frac{1}{3} k^{i} k_{k} n_{m} q^{k} q_{x x x}^{m}+\frac{2}{3} k^{i} k_{k} n_{m} q_{x}^{k} q_{x x}^{m} \\
& \quad+\frac{2}{3} k^{i} k_{k} k_{m} k_{j}(b . n) q^{j} q^{m} q_{x}^{k}+\frac{1}{3} k^{i} k_{n} n_{k} n_{m} q^{k} q^{m} q_{x}^{n}+\frac{1}{3} b^{i} k_{k} k_{m} n_{n} q^{k} q^{m} q_{x}^{n} .
\end{aligned}
$$

We have some particular solutions of Eqs. (30)-(32).

(i) For $N=2$ we have

$$
b_{j}^{i}=\delta_{j}^{i}-y^{i} y_{j}=x^{i} x_{j}, \quad s_{j k}^{i}=\frac{3}{2} \alpha_{1} x^{i} x_{j} x_{k}+\alpha_{2} x^{i} y_{j} y_{k}+\frac{1}{2} \alpha_{1} y^{i}\left(y_{j} x_{k}+y_{k} x_{j}\right),
$$

where $i, j=1,2$ and

$$
x^{i}=\delta_{1}^{i}, \quad y^{i}=\delta_{2}^{i},
$$

and

$$
k_{i}=y_{i}, \quad n_{i}=\frac{1}{2} \alpha_{1} x_{i}, \quad b_{i}=\alpha_{2} x_{i}
$$

The constants $a_{j k}^{i}$ and $c_{j k}^{i}$ appearing in the recursion operator are given by

$$
a_{j k}^{i}=\alpha_{1} x^{i} x_{j} x_{k}+\alpha_{2} x^{i} y_{j} y_{k}+\frac{1}{2} \alpha_{1} y^{i} x_{j} y_{k}, \quad c_{j k}^{i}=\frac{1}{2} \alpha_{1} x^{i} x_{j} x_{k}+\frac{1}{2} \alpha_{1} y^{i} x_{j} y_{k} .
$$

Taking $\alpha_{1}=2$ and $\alpha_{2}=1$ (without loss of generality), we obtain the following coupled system,

$$
\begin{aligned}
& u_{t}=u_{x x x}+3 u u_{x}+v v_{x}, \\
& v_{t}=(u v)_{x} .
\end{aligned}
$$

The above system was first introduced by Ito [3] and the bi-Hamiltonian structure has been studied by Olver and Rosenau [4]. The recursion operator of this system is given by

$$
R=\left(\begin{array}{cc}
D^{2}+2 u+u_{x} D^{-1} & v \\
v+v_{x} D^{-1} & 0
\end{array}\right)
$$

The first generalised symmetry of the system is found as

$$
\begin{aligned}
& \frac{\partial u}{\partial \tau}=u_{x x x x x}+5 u u_{x x x}+v v_{x x x}+10 u_{x} u_{x x}+3 v_{x} v_{x x}+3 u v v_{x}+\frac{15}{2} u^{2} u_{x}+\frac{3}{2} v^{2} u_{x}, \\
& \frac{\partial v}{\partial \tau}=v u_{x x x}+3 u v u_{x}+\frac{3}{2} v^{2} v_{x}+v_{x} u_{x x}+\frac{3}{2} u^{2} v_{x} .
\end{aligned}
$$


(ii) For $N=3$, we have $b_{j}^{i}=\delta_{j}^{i}-z^{i} z_{j}=x^{i} x_{j}+y^{i} y_{j}$, where $i, j, k=1,2,3$ and $s_{j k}^{i}$ are found as

$$
\begin{aligned}
s_{j k}^{i} & =x^{i}\left[3 \alpha_{0} x_{j} x_{k}+3 \alpha_{1}\left(x_{k} y_{j}+x_{j} y_{k}\right)+3 \alpha_{2} y_{j} y_{k}+\beta_{1} z_{j} z_{k}\right]+y^{i}\left[3 \alpha_{3} x_{j} x_{k}+3 \alpha_{4}\left(x_{k} y_{j}+x_{j} y_{k}\right)\right. \\
& \left.+3 \alpha_{5} y_{j} y_{k}+\beta_{2} z_{j} z_{k}\right]+z^{i}\left[\alpha_{6}\left(x_{j} z_{k}+x_{k} z_{j}\right)+\alpha_{7}\left(y_{j} z_{k}+y_{k} z_{j}\right)\right],
\end{aligned}
$$

where for simplicity we have taken

$$
x^{i}=\delta_{1}^{i}, \quad y^{\prime}=\delta_{2}^{i}, \quad z^{i}=\delta_{3}^{i},
$$

and

$$
k_{i}=z_{i}, \quad n_{i}=\alpha_{6} x_{i}+\alpha_{7} y_{i}, \quad b_{i}=\beta_{1} x_{i}+\beta_{2} y_{i},
$$

and the constants $\left(\alpha_{0}, \ldots, \alpha_{7}\right)$ and $\left(\beta_{1}, \beta_{2}, \beta_{3}\right)$ have the following relations,

$$
\begin{array}{ll}
\alpha_{2}=\frac{\alpha_{1}\left(\alpha_{0} \alpha_{6}+\alpha_{1} \alpha_{3}-\alpha_{6}^{2}\right)}{\alpha_{3}\left(\alpha_{0}-\alpha_{6}\right)}, & \beta_{3}=\alpha_{6}, \quad \alpha_{4}=\frac{\alpha_{2} \alpha_{3}}{\alpha_{1}}, \\
\alpha_{5}=\frac{-\alpha_{0} \alpha_{1} \alpha_{2}+\alpha_{1}^{3}+\alpha_{2}^{2} \alpha_{3}}{\alpha_{1}^{2}}, & \alpha_{7}=\frac{-\alpha_{0} \alpha_{2}+\alpha_{1}^{2}+\alpha_{2} \beta_{3}}{\alpha_{1}}, \quad \beta_{2}=\frac{\beta_{1}\left(\beta_{3}-\alpha_{0}\right)}{\alpha_{1}} .
\end{array}
$$

The constants $c_{j k}^{i}$ are given by

$$
\begin{aligned}
c_{j k}^{i} & =x^{i}\left[\alpha_{0} x_{i} x_{k}+\alpha_{1}\left(x_{k} y_{j}+x_{j} y_{k}\right)+\alpha_{2} y_{j} y_{k}\right] \\
& +y^{i}\left[\alpha_{3} x_{j} x_{k}+\alpha_{4}\left(x_{k} y_{j}+x_{j} y_{k}\right)+\alpha_{5} y_{j} y_{k}\right]+z^{i}\left(\alpha_{6} x_{j} z_{k}+\alpha_{7} y_{j} z_{k}\right),
\end{aligned}
$$

and $a_{j k}^{i}=s_{k, j}^{i}-c_{k, j}^{i}$. Letting $q^{i}=(u, v, w)$, the system integrable equations for $u, v$ and $w$ are found as

$$
\begin{aligned}
& u_{t}=u_{x x x}+3 \alpha_{0} u u_{x}+3 \alpha_{1}(u v)_{x}+\beta_{1} w w_{x}+3 \alpha_{2} v v_{x}, \\
& v_{1}=v_{x x x}+3 \alpha_{3} u u_{x}+3 \alpha_{4}(u v)_{x}+3 \alpha_{5} v v_{x}+\beta_{2} w w_{x}, \\
& w_{t}=\alpha_{6}(u w)_{x}+\alpha_{7}(v w)_{x},
\end{aligned}
$$

and the recursion operator is given by

$$
R=\left(\begin{array}{ccc}
D^{2}+2 \alpha_{0} u+2 \alpha_{1} v+r_{0} D^{-1} & 2 \alpha_{1} u+2 \alpha_{2} v+r_{1} D^{-1} & \beta_{1} w \\
2 \alpha_{3} u+2 \alpha_{4} v+r_{2} D^{-1} & D^{2}+2 \alpha_{4} u+2 \alpha_{5} v+r_{3} D^{-1} & \beta_{2} w \\
\alpha_{6}\left(w+w_{x} D^{-1}\right) & \alpha_{7}\left(w+w_{x} D^{-1}\right) & 0
\end{array}\right),
$$

where

$$
r_{0}=\alpha_{0} u_{x}+\alpha_{1} v_{x}, \quad r_{1}=\alpha_{1} u_{x}+\alpha_{2} v_{x}, \quad r_{2}=\alpha_{3} u_{x}+\alpha_{4} v_{x}, \quad r_{3}=\alpha_{4} u_{x}+\alpha_{5} v_{x} .
$$

The first generalised symmetry of the system (49)-(51) as an expression is too long, hence we do not give it here.

As a summary, we have found infinitely many integrable systems of nonlinear partial differential equations corresponding to each value of $N$. We have also given the recursion operator of each system. In this work we took the rank of the matrix $b_{j}^{i}$ as $N-1$. It is also possible to have integrable systems of nonlinear partial differential equations with lower rank $b_{j}^{i}$,s. In the general case with arbitrary rank we have

$$
\begin{aligned}
& q_{i}^{\mu_{1}}=q_{x x x}^{\mu_{1}}+s_{i j}^{\mu_{1}} q^{i} q_{x}^{j} \\
& q_{1}^{\mu_{2}}=s_{i j}^{\mu_{2}} q^{i} q_{x}^{j},
\end{aligned}
$$


where $\mu_{1}=1,2, \ldots, r, \mu_{2}=r, r+1, \ldots, N$ and $r$ is the rank of the matrix $b_{j}^{i}$. We have found the constants $a_{j k}^{i}, c_{j k}^{i}$ for $r=N-1$. For other values of $r$ these constants will be different from those given in (24) and (25). These systems and a detailed discussion of this work will be published elsewhere.

We thank Maxim Pavlov for discussions on the Svinolupov systems. This work is partially supported by the Scientific and Technical Research Council of Turkey (TUBITAK). M.G is an associate member of the Turkish Academy of Sciences (TUBA).

\section{References}

[1] S.I. Svinolupov, Theor. Mat. Fiz. 87 (1991) 391.

|2| S.I. Svinolupov, Functional Anal. Appl. 27 (1994) 257.

|3| M. Ito, Phys. Lett. A 91 (1982) 335.

14| P.J. Olver and P. Rosenau, Tri-Hamiltonian soliton-compacton duality, preprint (4 April 1995).

$15 \mid \ddot{O}$. Oguz, A canonical Lagrangian formalism of multicomponent KdV equations, preprint (1995). 